\section{Conhecimento e voluntariedade para participação em pesquisas: um estudo descritivo com participantes de um ensaio clínico}

\author{
Knowledge and willingness to participate in \\ research: a descriptive study of volunteers \\ in a clinical trial
}

\section{Conocimiento y la voluntariedad de participar en la investigación: un estudio descriptivo con los participantes en un ensayo clínico}

\begin{abstract}
The aim of this study was to evaluate volunteers' knowledge of the information on the free informed consent form and their willingness to participate in a clinical trial. This was a quantitative, descriptive, cross-sectional study conducted in November 2011 with subjects from a clinical trial in Americaninhas, northeast Minas Gerais State, Brazil. A convenience sample in cluded 143 adults of both sexes, 18 to 45 years of age. A structured questionnaire was applied one week after signing the free informed consent form. Most participants signed the free informed consent without sufficient knowledge of the research information and were influenced in their decision to participate in the trial. The authors conclude that signing the free informed consent form fails to express all participants' autonomy in clinical trials.
\end{abstract}

Research Ethics; Clinical Trial; Informed Consent; Personal Autonomy
Lucas Lobato 1

Maria Flávia Gazzinelli 1

Andréa Gazzinelli 1

Amanda Nathale Soares 1

\section{Resumo}

O objetivo deste estudo é avaliar o conhecimento sobre as informações do Termo de Consentimento Livre e Esclarecido (TCLE) e a voluntariedade de participantes de um ensaio clínico. Trata-se de um estudo quantitativo, descritivo, transversal, realizado em novembro de 2011 com participantes de um ensaio clínico realizado em Americaninhas, no Nordeste de Minas Gerais, Brasil. Amostra por conveniência, com 143 adultos de 18 a 45 anos, de ambos os sexos. Utilizou-se um questionário estruturado, aplicado uma semana após a assinatura do TCLE. A maioria dos participantes do ensaio clínico assinou o TCLE sem o conhecimento suficiente das informações da pesquisa, e sofreu influência em sua decisão de participar do ensaio clínico. Concluímos que a assinatura do TCLE não garante a expressão da autonomia de todos os participantes de pesquisa clínica.

Ética em Pesquisa; Ensaio Clínico; Consentimento Livre e Esclarecido; Autonomia Pessoal 


\section{Introdução}

O Consentimento Livre e Esclarecido (CLE) é respeitado como um dos princípios éticos fundamentais na condução de pesquisas com seres humanos 1. Possui o objetivo de tratar o participante de pesquisa em sua dignidade, por meio da promoção e da proteção desse direito ${ }^{2}$. No Brasil, além do valor ético, o CLE é considerado uma requisição legal, materializada na assinatura do Termo de Consentimento Livre e Esclarecido (TCLE), dispositivo que possibilita a expressão do princípio da autonomia ${ }^{3}$.

Para que o CLE expresse uma ação autônoma é necessário que duas condições sejam cumpridas pelo voluntário de pesquisa ao assinar um TCLE: compreensão pormenorizada sobre as informações da pesquisa e decisão voluntária 4 . O cumprimento dessas condições está associado a fatores que favorecem a expressão da autonomia do participante, como o maior controle do sujeito sobre sua ação, a autenticidade em sua decisão, a menor chance de arrependimento da decisão tomada e a menor ansiedade no processo decisório $5,6,7$.

Nas últimas décadas, vem sendo questionada a capacidade do TCLE de garantir a expressão de uma ação autônoma para todos os participantes de pesquisa, sobretudo em pesquisas clínicas. Essas pesquisas envolvem riscos que podem afetar a saúde de seus participantes, exigindo rigorosamente uma decisão autônoma ${ }^{8}$. No entanto, frequentemente, voluntários de ensaios clínicos assinam esse documento com baixo conhecimento sobre as informações do estudo 9,10,11,12 e a voluntariedade é afetada por influências em sua decisão 13,14.

Esse questionamento torna-se maior em pesquisas clínicas realizadas em países em desenvolvimento. Nesses países, comumente se evidenciam características que podem prejudicar a compreensão das informações da pesquisa e a voluntariedade desses participantes, como as precárias condições socioeconômicas 15,16,17 e as múltiplas interpretações de conceitos científicos, como: "voluntariedade", "randomização" e "benefícios e riscos” 18,19. Diante dessas características, os participantes de pesquisas clínicas realizadas em países em desenvolvimento podem estar sujeitos à exploração de seus direitos 20,21,22.

No Brasil, um terço da população vive em condições de extrema pobreza, dilatada parcela de sua população é analfabeta funcional ou possui baixo nível de educação formal 23 , e participantes de pesquisas desconhecem conceitos metodológicos 24 . Nesse contexto, questionamos com este estudo se em uma pesquisa clínica realizada no Brasil a assinatura do TCLE é capaz de expressar uma ação autônoma para todos os participantes.

Para responder a essa questão nós avaliamos o conhecimento e a voluntariedade de participantes de um ensaio clínico após a assinatura do TCLE. Esse ensaio possuía o objetivo de avaliar a tolerabilidade de um alimento funcional, com qualidades anti-helmínticas, em adultos, e era intitulado Estudo Duplo-Cego, Randomizado, Controlado da Tolerabilidade do Consumo Regular de uma Mistura de Óleos, em Adultos Residentes numa Área Endêmica para Helmintos (ABS-00-02). Sua realização ocorreu no Nordeste de Minas Gerais (Americaninhas e adjacências), distrito do Município de Novo Oriente de Minas, no período de outubro de 2011 a março de 2012. Perante o exposto, o objetivo deste estudo é avaliar o conhecimento sobre as informações do TCLE e a voluntariedade de participantes de um ensaio clínico.

No Brasil, onde os direitos do participante de pesquisa são explicitamente protegidos 3 , torna-se indispensável estudar se o TCLE é capaz de expressar uma decisão autônoma de todos participantes de ensaios clínicos, tendo em vista que falhas no processo de obtenção do Termo podem resultar em violações dos direitos dos participantes. Este estudo também é importante devido ao aumento do número de pesquisas clínicas realizadas no Brasil e na América Latina nas últimas décadas 25 . No entanto, os estudos nacionais estão concentrados na avaliação da qualidade na prática clínica 25,26 e na análise de legibilidade do TCLE 27, tendo pouca atenção destinada à qualidade deste documento na participação em ensaios clínicos 28,29.

\section{Método}

Trata-se de um estudo descritivo, com configuração transversal e abordagem quantitativa, desenvolvido com os participantes do ensaio clínico ABS-00-02. Esse estudo foi realizado em Americaninhas, distrito do Município de Novo Oriente de Minas, localizado na mesorregião do Vale do Mucuri, Nordeste de Minas Gerais, Brasil O Distrito de Americaninhas localiza-se a $60 \mathrm{~km}$ de Novo Oriente de Minas e a 556km da capital Belo Horizonte. O referido ensaio clínico foi realizado por um consórcio de pesquisadores da Universidade George Washington (Washington DC, Estados Unidos), em parceria com o Centro de Pesquisas René Rachou, Fundação Oswaldo Cruz (Fiocruz).

Inicialmente, a disposição dos potenciais voluntários para participar do ensaio clínico ABS-00-02 foi avaliada por meio de uma reunião 
coletiva em que foram dadas as informações referentes a esta investigação. Esses voluntários foram convidados a participar dessa reunião de acordo com os critérios previamente adotados para o ensaio clínico, quais sejam: idade entre 18-45 anos e residir no Município de Novo Oriente de Minas. Ao todo foram recrutados 150 potenciais participantes para o ensaio clínico, os quais se voluntariaram prontamente para essa investigação quando foram convidados. Nessa reunião compareceram 143 participantes, para os quais foi agendada a realização do consentimento individual.

O processo de consentimento para o ensaio clínico ABS-00-02 foi então realizado individualmente, no domicílio dos participantes, por enfermeiros responsáveis pela equipe clínica dessa investigação. Nessa visita domiciliar o potencial participante da investigação clínica foi convidado a ler o TCLE. Em caso de desejo do voluntário de que a leitura fosse realizada por um membro da equipe de pesquisa, esse documento era lido em voz alta, juntamente a uma testemunha indicada pelo voluntário. Se o participante fosse analfabeto, solicitava-se a indicação de uma pessoa de sua confiança, habilitada para leitura, para que fosse testemunha do processo de consentimento. Após esse processo, era realizada uma discussão com o membro da equipe sobre as possíveis dúvidas. Todos os participantes foram encorajados a esclarecer dúvidas que porventura surgiriam durante esse processo. Ao final, em caso de positividade para a participação nessa investigação clínica, pedia-se que o TCLE fosse assinado.

Ao final desse processo, o ensaio clínico ABS-00-02 recrutou 143 participantes, tamanho amostral previamente estabelecido para o trabalho, uma vez que este valor considerou a perda amostral. Todos os voluntários da investigação clínica aceitaram participar deste estudo sobre a avaliação do conhecimento sobre as informações do TCLE e a voluntariedade de participantes de um ensaio clínico. Esses participantes tinham média de idade de 34,2 anos (DP: 7,14), eram em sua maioria do sexo feminino $(67,6 \%)$, pardo/ negro $(77,7 \%)$, com o ensino fundamental ou analfabeto $(65,43 \%)$, casados $(79,7 \%)$ e com renda familiar superior a R\$ 545,00 (50,7\%) - salário mínimo vigente na data do estudo de acordo com a Lei no 12.38230 .

Os voluntários preencheram um questionário estruturado, autoaplicável, que avaliou o conhecimento sobre as informações do ensaio clínico e a voluntariedade na decisão de participar da investigação clínica.

Para a avaliação do conhecimento foram elaboradas 12 questões abertas que abordavam as informações necessárias para o CLE do participante de pesquisa, segundo as Diretrizes $e$ Normas Regulamentadoras de Pesquisas Envolvendo Seres Humanos ${ }^{3}$. Essas informações avaliavam o conhecimento sobre os métodos e os objetivos da pesquisa, os direitos do participante, o patrocinador do estudo, os benefícios e os riscos da pesquisa. Optou-se por questões abertas para se obter maior fidedignidade dos dados, haja vista que as respostas pré-determinadas de questões fechadas podem influenciar as respostas dos participantes ${ }^{31}$, e que o conhecimento deles sobre as informações do TCLE foi superestimado quando avaliado por instrumentos que utilizavam questões fechadas. Na elaboração das questões optou-se pela utilização de linguagem clara e de fácil entendimento (avaliados pelo Índice de Facilidade de Leitura de Flesch), por termos de conhecimento geral ao contrário de termos técnicos e pela não utilização de frases negativas e de palavras de significado duplo 31. A voluntariedade foi avaliada pela identificação de diferentes fatores de influência na tomada de decisão para participar do ensaio clínico. Essa avaliação foi embasada no conceito de que "a voluntariedade é a possibilidade que a pessoa tem de tomar decisões sem ser constrangida ou coagida para que decida por uma das alternativas - construída ao longo do desenrolar do processo de consentimento" 2 (p. 81).

As entrevistas foram realizadas uma semana após a assinatura do TCLE do ensaio clínico (novembro de 2011) por meio de um questionário estruturado. Esse questionário foi testado em um estudo piloto para a adequação aos participantes e baseado em outros estudos realizados em Americaninhas 28. A aplicação desse instrumento durou, em média, 10 minutos e foi realizada no domicílio do entrevistado, sem a interferência de terceiros ou de ruídos que comprometessem a realização do levantamento ou que desviassem a atenção do participante.

Para assegurar a confiabilidade foi realizada a dupla entrada independente dos dados. Quando houve discordância, o caso assinalado foi conferido no questionário original e corrigido. Os resultados foram tabelados e analisados pelo software SPSS 14 (SPSS Inc., Chicago, Estados Unidos). As questões sobre o conhecimento das informações do TCLE e os fatores influentes na decisão dos participantes foram analisados pela frequência relativa e absoluta. Posteriormente, foi calculado o somatório de acerto de todas as questões que avaliaram o conhecimento sobre o TCLE, bem como dos fatores que influenciaram a decisão dos voluntários. Consideramos um percentual de acerto acima de $75 \%$ das informações do TCLE como conhecimento satisfatório das in- 
formações deste documento para uma decisão autônoma ${ }^{9}$ e nenhuma influência na decisão do participante como uma decisão voluntária. Esses resultados foram expressos por meio de frequência relativa e absoluta, e por média aritmética e desvio padrão.

Com base na conceituação amplamente aceita de autonomia 2, em que são necessárias para o participante informações substanciais sobre a pesquisa e voluntariedade em sua decisão, considerou-se, para este estudo, uma decisão autônoma aquela que o participante da pesquisa atingiu, ao mesmo tempo, um percentual de acerto igual ou superior a $75 \%$ e nenhuma influência em sua decisão de participar do estudo clínico.

Opresente estudo foi aprovado pelo Comitêde Ética em Pesquisa da Universidade Federal de Minas Gerais (protocolo no 01743412.3.0000.5149). Todas as normas e diretrizes brasileiras ${ }^{3}$ para a condução de pesquisas com seres humanos foram rigorosamente seguidas pelos pesquisadores no processo de consentimento do ensaio clínico ABS-00-02 e do presente estudo.

\section{Resultados}

O conhecimento médio dos participantes sobre as informações do TCLE foi de 56,16\% com desvio padrão de $18,35 \%$. A maioria dos participantes conhecia os exames realizados no ensaio clínico ABS-00-02 (95,10\%), o direito de se retirar da investigação clínica (93,00\%), a ausência de ônus financeiro $(91,60 \%)$, as alternativas de assistência médica além da oferecida pelo ensaio clínico $(79,72 \%)$, os benefícios $(75,52 \%)$ e o direito à confidencialidade dos dados $(72,72 \%)$. Em contrapartida, a minoria dos participantes conhecia o patrocinador $(18,20 \%)$, a duração do ensaio clínico $(18,20 \%)$, o motivo pelo qual foi convidado a participar da investigação clínica (32,36\%), o objetivo da investigação (48,95\%), os efeitos adversos $(45,45 \%)$ e que a desistência de participar da pesquisa não envolveria a perda da assistência médica (32,86\%) (Tabela 1).

Em relação aos fatores que influenciaram a decisão dos voluntários, observamos que o pesquisador, o tratamento médico e as reuniões com os pesquisadores influenciaram as decisões. Em contraponto, os amigos, a família e a possibilidade de realizar exames médicos apareceram como fatores que apresentaram menores influências (Tabela 2).

A Tabela 3 detalha o somatório dos fatores influentes na decisão dos participantes do ensaio clínico. Observamos que a maior parte deles tem pelo menos um fator influente em sua decisão
Tabela 1

Frequências absoluta e relativa de acertos dos participantes sobre as informações do ensaio clínico ABS-00-02. Distrito de Americaninhas, Município de Novo Oriente de Minas, Minas Gerais, Brasil, 2011 ( $n=143$ ).

\begin{tabular}{lcc}
\hline Questão & $\mathbf{n}$ & $\%$ \\
\hline Patrocinador & 26 & 18,20 \\
Tempo de duração & 26 & 18,20 \\
Motivo do convite & 47 & 32,86 \\
Efeitos adversos & 65 & 45,45 \\
Objetivo & 70 & 48,95 \\
Benefícios & 108 & 75,52 \\
Exames e procedimentos & 136 & 95,10 \\
Perda do tratamento & 47 & 32,86 \\
Sigilo e confidencialidade & 104 & 72,72 \\
Remuneração & 131 & 91,60 \\
Alternativas de tratamento & 114 & 79,72 \\
Retirar-se da pesquisa & 133 & 93,00 \\
\hline
\end{tabular}

Tabela 2

Fatores influentes na decisão dos participantes do ensaio clínico ABS-00-02. Distrito de Americaninhas, Município de Novo Oriente de Minas, Minas Gerais, Brasil, 2011 ( $n=143$ ).

\begin{tabular}{lcl}
\hline Fatores influentes & $\mathbf{n}$ & \% \\
\hline Tratamento médico & 131 & 92 \\
Pesquisador & 126 & 88 \\
Reuniões & 124 & 87 \\
Exames & 100 & 70 \\
Família (cônjuge) & 89 & 62 \\
Amigos & 79 & 55 \\
\hline
\end{tabular}

Tabela 3

Frequências relativa e absoluta do número de influências na decisão de participantes do ensaio clínico ABS-00-02. Distrito de Americaninhas, Município de Novo Oriente de Minas, Minas Gerais, Brasil, 2011 ( $n=143$ ).

\begin{tabular}{lll}
\hline Número de influências & $\mathbf{n}$ & $\%$ \\
\hline Nenhuma (zero) * & 37 & 25,9 \\
1 & 30 & 21,0 \\
2 & 32 & 22,4 \\
3 & 23 & 16,1 \\
4 & 10 & 7,0 \\
5 & 6 & 4,2 \\
6 & 5 & 3,5 \\
\hline
\end{tabular}

* Decisão voluntária. 
para participar do ensaio clínico $(74,1 \%)$, e o restante dos participantes realizou uma decisão sem influências, ou seja, uma decisão voluntária.

A Tabela 4 descreve o porcentual de participantes que tinham conhecimento adequado das informações do TCLE e/ou realizaram uma decisão voluntária. Observamos que 20,3\% deles conheciam adequadamente as informações do TCLE, e 28\% não sofreram pelo menos uma influência em sua decisão. A maioria dos participantes $(57,3 \%)$ assinou o TCLE sem o conhecimento das informações presentes no documento e sob a influência de, ao menos, um fator em sua decisão, e 5,6\% dos participantes realizaram uma decisão autônoma.

\section{Discussão}

Os resultados deste estudo evidenciaram que a maioria dos participantes do ensaio clínico ABS-00-02 assinou o TCLE sem o conhecimento suficiente das informações da pesquisa e com influência em sua decisão. Essa conclusão é apoiada na afirmação, consensual na literatura, de que uma decisão é considerada autônoma quando o participante de uma pesquisa compreende as informações e decide voluntariamente sobre a sua participação 2,3,4.

A constatação dessa insuficiência é importante para se refletir que essa situação pode ser evidenciada em outros tipos de pesquisa no Brasil, uma vez que neste país o TCLE pode ser adotado pelo pesquisador como o único meio de divulgação das informações do estudo ao participante da pesquisa $3 \mathrm{~A}$ insuficiência desse documento também aponta para a necessidade de elaborar e aplicar estratégias que busquem assegurar uma decisão autônoma pelos participantes de pesquisas. Dentre essas estratégias destacamos a associação de uma intervenção educativa com os potenciais participantes antes da assina- tura do TCLE. Essa estratégia vem se mostrando a mais eficaz para a melhoria do conhecimento das informações da pesquisa 32 e para o favorecimento de decisões voluntárias 29.

Os resultados deste estudo demonstram que, assim como em outros países 15,31,33,34,35, o TCLE é insuficiente para expressar uma decisão autônoma, sugerindo que este é um desafio ético para a condução de pesquisas com seres humanos. Esse desafio pode ser ainda maior em pesquisas clínicas realizadas no Brasil, uma vez que os resultados deste estudo mostraram que o conhecimento médio sobre as informações do TCLE e o porcentual de respondentes que possui conhecimento suficiente sobre esse documento foi inferior ao evidenciado em outras pesquisas clínicas, inclusive em estudos realizados em países em desenvolvimento 9,11,15,16.

Esse resultado pode estar associado ao fato de muitos participantes do ensaio clínico ABS00-02 assinarem o TCLE sem questionarem suas dúvidas acerca das informações do documento, situação apoiada por um ensaio clínico desenvolvido na África do Sul, em que $40 \%$ dos participantes consentiram em participar da investigação mesmo com dúvidas sobre o TCLE 36 . Essa explicação está amparada na prevalência de participantes de menor escolaridade e de baixo nível socioeconômico, características associadas à menor capacidade de formular perguntas e à menor confiança para realizá-las durante o processo de obtenção do TCLE 37 . A não realização de perguntas também pode ser explicada pela sobreposição da imagem do médico à do pesquisador evidenciada na região deste estudo 38 , tendo em vista que a autoridade do médico pode inibir o questionamento sobre as condutas adotadas por este profissional 11,39.

A ação autônoma pode ser prejudicada não só pelo conhecimento inadequado das informações do TCLE, mas também pelo desconhecimento de informações específicas do documento. Neste

Tabela 4

Conhecimento sobre as informações do TCLE e influência na decisão dos participantes do ensaio clínico. Distrito de Americaninhas, Município de Novo Oriente de Minas, Minas Gerais, Brasil, 2011.

\begin{tabular}{lccc}
\hline Conhecimento sobre as informações do TCLE & \multicolumn{2}{c}{ Influência } & Sim \\
& Não & $21(14,7 \%)$ & $29(20,3 \%)$ \\
Adequado & $8(5,6 \%)^{*}$ & $82(57,3 \%)$ & $114(79,7 \%)$ \\
Inadequado & $32(22,4 \%)$ & $103(72,0 \%)$ & $143(100,0 \%)$ \\
Total & $40(28,0 \%)$ & 100,0 \\
\hline
\end{tabular}

* Decisão autônoma. 
estudo, por exemplo, os resultados demonstraram que a maioria dos participantes desconhecia o objetivo da pesquisa clínica e os seus efeitos adversos. A implicação ética do desconhecimento desses temas repousa na possibilidade de manifestação do "equívoco terapêutico", fenômeno em que participantes de pesquisas clínicas não distinguem o tratamento médico do objetivo da investigação científica e, desta forma, acreditam que os protocolos de pesquisa foram desenhados para o seu interesse 40 .

O Equívoco Terapêutico é definido como a fusão entre o objetivo científico de uma pesquisa clínica e a finalidade do cuidado médico 41 , e sua causa está associada ao desconhecimento da natureza de uma pesquisa clínica e dos procedimentos metodológicos presentes nesse tipo de estudo 42,43 . Sabe-se que a manifestação desse fenômeno está em contraste com a doutrina do CLE, uma vez que o participante de pesquisa que padece desses equívocos pode falhar na avaliação dos riscos e dos benefícios de sua participação na pesquisa clínica e fundamentar sua decisão em critérios incorretos e em falsas esperanças 44 .

A manifestação desse fenômeno é reforçada por um estudo realizado em Americaninhas, em que os participantes de um ensaio clínico para teste de uma vacina contra ancilostomíase acreditaram que o objetivo da investigação era o tratamento de seus participantes ${ }^{38}$. Essa problemática, novamente, aproxima os resultados desta pesquisa com os obstáculos presentes em pesquisas realizadas nos países em desenvolvimento, uma vez que em outros ensaios clínicos realizados nesses países também se observou desconhecimento desses temas 12,45,46.

Por outro lado, uma novidade revelada por este estudo é o conhecimento de praticamente todos os participantes sobre o direito de se retirar da investigação clínica ABS-00-02. A importância desse achado reside em sua exclusividade evidenciada em países em desenvolvimento, uma vez que em pesquisas realizadas nesses países comumente se observou baixo conhecimento sobre esse direito 12. Em termos éticos, esse conhecimento que os participantes mostraram possuir é fundamental para a voluntariedade de sua decisão e, portanto, para uma decisão autônoma.

O conhecimento desse direito, no entanto, pode não ser suficiente para que o participante possa se retirar de um ensaio clínico de acordo com o seu interesse. Isso porque em países em desenvolvimento, não raro, esse direito pode ser comprometido pela ausência de alternativas ao tratamento médico além da participação em uma pesquisa clínica. Por não vislumbrar alternativas de acesso à saúde, o participante de pes- quisa clínica sente-se compelido a participar ou a não abandoná-la 47 .

Isso pode ser destacado no presente estudo, uma vez que a maioria dos participantes desconhecia o direito de continuar recebendo a assistência médica oferecida pelo ensaio clínico, situação também observada em outros estudos realizados em países em desenvolvimento 48,49 . No entanto, a maioria dos participantes conhecia as alternativas para assistência médica, além da participação no ensaio clínico ABS-00-02, o que pode reduzir a influência do acesso à saúde na decisão de permanecer no estudo.

Sobre a importância do conhecimento dos participantes acerca das informações contidas no TCLE, destaca-se o conceito de Letramento em Saúde (do inglês health literacy), cuja definição ainda não apresenta consenso na literatura 50. O Letramento em Saúde pode ser definido como as "competências cognitivas e sociais que determinam a motivação e a capacidade dos indivíduos para obter acesso, compreender e utilizar a informação em meios que promovem e mantêm uma boa saúde" 51 (p. 10). Pode ser também definido como "o grau pelo qual os indivíduos têm a capacidade para obter, processar e entender informações básicas de saúde e serviços necessários para a tomada de decisões adequadas em saú$d e " 52$ (p. 147; tradução livre dos autores).

Nesse sentido, ressalta-se que, para melhor compreensão das informações e para a tomada de decisões mais autônomas, atenção especial deve ser destinada à linguagem dos textos relativos ao campo da saúde, com destaque aqui para o TCLE. Essa necessária atenção sustenta-se no fato de que mesmo um nível educacional elevado pode ser insuficiente para que os sujeitos possam compreender os significados das linguagens médica e técnica 50 .

Um importante achado deste estudo é a identificação de que a maioria dos partícipes do ensaio clínico sofreu ao menos uma influência em sua decisão para se inscrever na pesquisa. Esse achado destaca-se na literatura sobre o tema, considerando que estudos empíricos apontam ou sugerem a presença dessas influências, mas sem analisar o seu grau na decisão dos participantes de pesquisas 39,44. Essa descoberta é importante ao consideramos que essas influências têm um aspecto gradual, assumindo uma relação inversamente proporcional à autonomia na decisão desses participantes 2 .

Outro relevante achado deste estudo é o fato de que a família ou o cônjuge representou as menores influências na decisão de participar no ensaio clínico ABS-00-02. Isso porque esperávamos uma maior influência desses fatores, tendo em vista que a maioria dos participantes dessa 
pesquisa era composta por mulheres casadas e que suas decisões poderiam ser afetadas pela estrutura familiar - pai ou marido -, como comumente é observado em países africanos 47,49 . A sustentação dessa presunção deu-se pelo fato de Americaninhas ser uma comunidade predominantemente rural, onde as formas de organizações familiares são estruturadas sobre a desigualdade de gênero e marcadas pela predominância da ótica de dominação masculina 49,53.

A principal influência sobre a decisão dos participantes do ensaio clínico ABS-00-02 foi o tratamento médico. Apesar de vários autores insistirem que esse fator é mais comum nos países em desenvolvimento 54 , estudos demonstram que ele é encontrado em sujeitos de estudos em todo o mundo 55,56, inclusive no Brasil 57. Como implicação ética dessa influência, destacase que a busca por tratamento médico pode ser um indício da precariedade de serviços de saúde disponíveis para a população, impelindo esses participantes a fazerem parte de pesquisas clínicas 13 .

A validade da conclusão deste estudo pode ser sustentada por diferentes estratégias metodológicas adotadas para minimizar a ocorrência de vieses nos dados coletados. Para evitar o viés de informação optamos pela utilização de perguntas abertas a fim de mensurar o conhecimento das informações do TCLE, uma vez que este formato é capaz de mensurar com maior precisão o conhecimento real sobre o tema, evitando que haja superestimação das respostas ou que estas sejam influenciadas por opções pré-determinadas 58. Também buscamos garantir que o conhecimento sobre esse tema fosse proveniente do processo de obtenção do TCLE e não de outras fontes, uma vez que aplicamos esse questionário uma semana após a assinatura do documento. A data de aplicação do questionário também evitou que o tempo entre a assinatura do TCLE e a coleta dos dados influenciasse o conhecimento sobre os temas avaliados 15 .

A validade dos dados foi também assegurada pela utilização da entrevista estruturada na aplicação do questionário. Isso porque esse tipo de coleta de dados assegura a padronização das entrevistas e das respostas obtidas, favorecendo a análise estatística dos dados e a sua confiabilidade. Para a garantia dessa padronização os entrevistadores foram devidamente treinados antes da aplicação do questionário, e as entrevistas foram realizadas individualmente, no domicílio dos participantes, evitando que fossem inibidos na declaração de suas respostas 31 .

O questionário utilizado neste estudo, embora não tenha sido validado, possui base teórica sólida sobre as recentes legislações nacionais, os regulamentos e as diretrizes sobre ética em pesquisa 59,60, e também teve por base a experiência dos pesquisadores em trabalhos semelhantes na mesma área.

\section{Conclusão}

Com base nos resultados deste estudo concluímos que a assinatura do TCLE não garante a expressão da autonomia para todos os participantes de uma pesquisa clínica. Concluímos também que as informações essenciais para uma decisão autônoma, como os objetivos do ensaio clínico, os seus benefícios e o direito à continuidade ao tratamento em caso de retirada do estudo são pouco conhecidas por esses participantes. Também é possível concluir que o tratamento médico oferecido pelo ensaio clínico foi o principal fator que influenciou a decisão do participante.

Em termos de pesquisa, sugere-se que seja realizado um estudo que avalie a possível relação entre voluntariedade e conhecimento das informações do TCLE. Em termos éticos, sugere-se a necessidade de avaliação constante da qualidade do CLE, especialmente o seu processo de obtenção. É de extrema importância a aproximação entre os pesquisadores e o Comitê de Ética em Pesquisa para que, juntos, possam elaborar o TCLE baseando-se em termos que esclareçam os sujeitos de pesquisa, tendo em vista, sobretudo, que os pesquisadores conhecem o contexto em que o trabalho será realizado. Também se faz necessária a iniciativa de desenvolver intervenções educativas capazes de criar circunstâncias para que o potencial participante de pesquisa forneça uma decisão autônoma. 


\section{Resumen}

El objetivo de este estudio es evaluar el conocimiento acerca de la información del Formulario de Consentimiento Informado y la disposición de los participantes para un ensayo clínico. Se trata de un análisis cuantitativo, descriptivo, de corte transversal, realizado en noviembre de 2011 con participantes de un ensayo clínico en Americaninhas, llevado a cabo en el nordeste de Minas Gerais, Brasil. Se trata de una muestra de conveniencia: 148 adultos entre 18 y 45 años, de ambos sexos. Se utilizó un cuestionario estructurado, cumplimentado una semana después de firmar el Formulario de Consentimiento Informado. La mayoría de los participantes del ensayo firmó el formulario de consentimiento informado sin un conocimiento suficiente de la investigación y fue influenciado en su decisión de participar en el ensayo clínico. Se concluye que la firma del formulario de consentimiento informado no garantiza la expresión de la autonomía de todos los participantes en la investigación clínica.

Ética en Investigación; Ensayo Clínico; Consentimiento Informado; Autonomía Personal

\section{Referências}

1. Emanuel EJ, Wendler D, Killen J, Grady C. What makes clinical research in developing countries ethical? The benchmarks of ethical research. J Infect Dis 2004; 189:930-7.

2. Junges JR. Exigências éticas do consentimento informado. Rev Bioét 2007; 15:78-89.

3. Ministério da Saúde; Conselho Nacional de Saúde; Comissão Nacional de Ética em Pesquisa. Resolução no 196, de 10 de outubro de 1996: diretrizes e normas regulamentadoras de pesquisa envolvendo seres humanos. http://conselho.saude.gov.br/ docs/Reso196.doc (acessado em 20/Jan/2013).

4. Beauchamp TL, Childress JF. Princípios de ética biomédica. 4a Ed. São Paulo: Edições Loyola; 2001.

5. Helgesson G, Ludvigsson J, Stolt UG. How to handle informed consent in longitudinal studies when participants have a limited understanding of the study. J Med Ethics 2005; 31:670-3.

6. Stryker JE, Wray RJ, Emmons KM, Winer E, Demetri G. Understanding the decisions of cancer clinical trial participants to enter research studies: factors associated with informed consent, patient satisfaction, and decisional regret. Patient Educ Couns 2006; 63:104-9.

7. Edwards SJF, Lilford RJ, Thornton J, Hewison J. Informed consent for clinical trials: in search of the best method. Soc Sci Med 1998; 47:1825-40.

\section{Colaboradores}

L. Lobato, M. F. Gazzinelli e A. Gazzinelli participaram da concepção e projeto, análise e interpretação dos dados, redação do artigo e revisão crítica relevante do conteúdo intelectual, e aprovação final da versão a ser publicada. A. N. Soares participou da análise e interpretação dos dados, redação do artigo, revisão crítica relevante do conteúdo intelectual, e aprovação final da versão a ser publicada.

\section{Agradecimentos}

Agradecemos a todos os voluntários do ensaio clínico ABS-00-02 e a todos os outros que diretamente ou indiretamente nos ajudaram na elaboração deste estudo.
8. Jansen LA. Two concepts of therapeutic optimism. J Med Ethics 2011; 37:563-6.

9. Minnies D, Hawkridge T, Hanekon W, Ehrlich R, London L, Hussey G. Evaluation of the quality of informed consent in a vaccine field trial in a developing country setting. BMC Med Ethics 2008; 9:1-9.

10. Cheng JD, Hilt J, Koczwara B, Schulman KA, Burnett CB, Gaskin JD, et al. Impact of quality of life on patient expectations regarding phase I clinical trials. J Clin Oncol 2000; 18:421-8.

11. Kaewpoonsri N, Okanurak K, Kitayaporn D, Kaewkungwa J, Vijaykadg S, Thamaree S. Factors related to volunteer comprehension of informed consent for a clinical trial. J Trop Med Public Health 2006; 37:996-1004.

12. Mandava A, Pace C, Campbell B, Grady C. The quality of informed consent: mapping the landscape. A review of empirical data from developing and developed countries. J Med Ethics 2012; 38:356-65.

13. Oduro AR, Aborigo RA, Amugsi D, Anto F, Anyorigiya T, Atuguba F, et al. Understanding and retention of the informed consent process among parents in rural Northern Ghana. BMC Med Ethics 2008; 9:1-9. 
14. Pace C, Emanuel EJ, Chuenyam T, Duncombe C, Bebchuk JD, Wendler D, et al. The quality of informed consent in a clinical research study in Thailand. IRB 2005; 27:9-17.

15. Chaisson LH, Kass NE, Chengeta B, Mathebula U, Samandari T. Repeated assessments of informed consent comprehension among HIV-infected participants of a three-year clinical trial in Botswana. PLoS One 2011; 6:e22.696.

16. Moodley K, Parker M, Myer L. Informed consent and participants perceptions of influenza vaccine trials in South Africa. J Med Ethics 2005; 31:727-32.

17. Lynöe N, Chowdhury M, Ekström L. Obtaining informed consent in Bangladesh. N Engl J Med 2002; 344:460-1.

18. Upvall M, Hashwani S. Negotiating the informed consent process in developing countries: a comparison of Swaziland and Pakistan. Int Nurs Rev 2001; 48:188-92.

19. Elbourne D, Snowdon C, Garcia J. Informed consent. Subjects may notunderstand concept of clinical trials. BMJ 1997; 315:248-9.

20. Cabral MML, Schindler HC, Abath FGC. Regulamentações, conflitos e ética da pesquisa médica em países em desenvolvimento. Rev Saúde Pública 2006; 40:521-7.

21. Scarpelli AC, Ferreira EF, Zarzar PMP. Vulnerabilidade socioeconômica versus autonomia na pesquisa em saúde. Rev Bioét 2007; 15:298-307.

22. Rogers W, Ballantyne A. Populações especiais: vulnerabilidade e proteção. RECIIS Revista Eletrônica de Comunicação, Informação e Inovação em Saúde 2008; 2 Suppl 1:1-41.

23. Instituto Paulo Montenegro. INAF Brasil 2009. Indicador de analfabetismo funcional: principais resultados. http://www.ipm.org.br/download/ inaf_brasil_2009_relatorio_divulgacao_revisto_ fev-11_vFinal.pdf (acessado em 12/Mar/2012).

24. Meneguin S, Zoboli ELCP, Domingues RZL, Nobre MR, Cesar LAM. Entendimento do termo de consentimento pelos pacientes partícipes em pesquisas com fármacos de cardiologia. Arq Bras Cardiol 2010; 94:4-9.

25. Glickman SW, McHutchison JG, Peterson ED, Cairns CB, Harrington RA, Califf RM, et al. Ethical and scientific implications of the globalization of clinical research. N Engl J Med 2009; 360:816-23.

26. Biondo-Simões MLP, Martynetz J, Ueda FMK, Olandosky M. Compreensão do termo de consentimento livre e esclarecido. Rev Col Bras Cir 2007; 34:183-8.

27. Miranda VC, Fêde ABS, Lera AT, Ueda A, Antomangelo DV, Brunetti K, et al. Como consentir sem entender? Rev Assoc Med Bras 2009; 55:328-34.

28. Gazzinelli MF, Lobato L, Matoso L, Avila R, Marques RC, Brown AS, et al. Health education through analogies: preparation of a community for clinical trials of a vaccine against hookworm in an endemic area of Brazil. PLoS Negl Trop Dis 2010; 4:749-62.

29. Lobato L, Souza V, Caçador B, Soares A, Wingester E, Gazzinelli M. Efeitos de intervenção educativa na qualidade ética do consentimento livre e esclarecido. Rev Bioét 2012; 20:479-89.
30. Brasil. Lei no 12.382, de 25 de fevereiro de 2011. Dispõe sobre o valor do salário mínimo em 2011 e a sua política de valorização de longo prazo; disciplina a representação fiscal para fins penais nos casos em que houve parcelamento do crédito tributário; altera a Lei no 9.430, de 27 de dezembro de 1996; e revoga a Lei no 12.255, de 15 de junho de 2010. Diário Oficial da União 2011; 28 fev.

31. Vieira S. Como elaborar questionários. São Paulo: Editora Atlas; 2009.

32. Flory J, Emanuel E. Interventions to improve research participants' understanding in informed consent for research a systematic review. JAMA 2004; 292:1593-601.

33. Joffe S, Cook EF, Cleary PD, Clark JW, Weeks JC. Quality of informed consent in cancer clinical trials: a cross-sectional survey. Lancet 2001; 358:1772-7.

34. Ellis RD, Sagara I, Durbin A, Dicko A, Shaffer D. Comparing the understanding of subjects receiving a candidate malaria vaccine in the United States and Mali. Am J Trop Med Hyg 2010; 83: 868-72.

35. Yuval R, Halon DA, Merdler A, Khader N, Karkabi $\mathrm{B}$, Uziel K, et al. Patient comprehension and reaction to participatingin a double blind randomized clinical trial (ISIS-4) in acute myocardial infarction. Arch Intern Med 2000; 160:1142-6.

36. Joubert G, Steinberg H, van der Ryst E, Chikobvu P. Consent for participation in the Bloemfontein vitamin a trial: how informed and voluntary? Am J Public Health 2003; 93:582-4.

37. Rajaraman D, Jesuraj N, Geiter L, Bennett S, Grewal HMS, Vaz M, et al. How participatory is parental consent in low literacy rural settings in low income countries? Lessons learned from a community based study of infants in South India Trials Study Group. BMC Med Ethics 2011; 12:1-9.

38. Karim Q, Karim S, Coovadia H, Susser M. Informed consent for HIV testing in a South African hospital: is it truly informed and truly voluntary? Am J Public Health 1998; 88:637-40.

39. Hespanha AC, Marques RC, Reis DC, Melo JMC, Diemert D, Gazzinelli MF. A pesquisa científica na saúde: uma análise sobre a participação de populações vulneráveis. Texto \& Contexto Enferm 2010; 19:104-11.

40. Lawrence DJ. The therapeutic misconception: not just for patients. J Can Chiropr Assoc 2008; 52: 139-42.

41. Henderson EG, Churchill LR, Davis AM, Easter MM, Grady C, Joffe S, et al. Clinical trials and medical care: defining the therapeutic misconception. PLoS Med 2007; 4:e324.

42. Appelbaum PS, Lidz CW, Grisso T. Therapeutic misconception in clinical research: frequency and risk factors. IRB 2004; 26:1-8.

43. Horng S, Grady C. Misunderstanding in clinical research: distinguishing therapeutic misconception, therapeutic misestimation, and therapeutic optimism. IRB 2003; 25:11-16.

44. Sreenivasan G. Informed consent and the therapeutic misconception: clarifying the challenge. J Clin Ethics 2005; 16:369-71. 
45. Manafa O, Lindegger G, Lisselmuiden C. Informed consent in an antiretroviral trial in Nigeria. Indian J Med Ethics 2007; 4:26-30.

46. Taiwo OO, Kass N. Post-consent assessment of dental subjects' understanding of informed consent in oral health research in Nigeria. BMC Med Ethics 2009; 10:1-7.

47. Mystakidou K, Panagiotou I, Katsaragakis S, Tsilika E, Parpa E. Ethical and practical challenges in implementing informed consent in HIV/AIDS clinical trials in developing or resource-limited countries. SAHARA J 2009; 6:46-57.

48. Tindana PO, Kass N, Akweongo P. The informed consent process in a rural African setting. IRB 2006; 28:1-6.

49. Ressel, LB, Gualda DMA. A sexualidade como uma construção cultural: reflexões sobre preconceitos e mitos inerentes a um grupo de mulheres rurais. Rev Esc Enferm USP 2003; 37:82-7.

50. Passamai MPB, Sampaio HAC, Dias AMI, Cabral LA. Functional health literacy: reflections and concepts on its impact on the interaction among users, professionals and the health system. Interface Comun Saúde Educ 2012; 16:301-14.

51. World Health Organization. Health promotion glossary. Geneva: World Health Organization; 1998.

52. Parker RM, Ratzan SC, Lurie N. Health literacy: a policy challenge for advancing high-quality health care. Health Aff 2003; 22:147-53.

53. Rocha-Coutinho ML. Dos contos de fada aos superheróis: mulheres e homens brasileiros reconfiguram identidades. Psicol Clín 2000; 2:65-82.
54. Shah JY, Phadtare A, Rajgor D, Vaghasia M, Pradhan S, Zelko H, et al. What leads Indians to participate in clinical trials? A meta-analysis of qualitative studies. PLoS One 2010; 5:e10730.

55. Stunkel L, Grady C. More than the money: a review of the literature examining healthy volunteer motivations. Contemp Clin Trials 2011; 32:342-52.

56. Kass N, Maman S, Atkinson J. Motivations, understanding and voluntariness in international randomized trials. IRB 2005; 27:1-8.

57. Nappo SA, Iafrate GB, Sanchez ZM. Motives for participating in a clinical research trial: a pilot study in Brazil. BMC Public Health 2013; 13:19.

58. Lindegger G, Milford C, Slack C, Quayle M, Xaba $\mathrm{X}$, Vardas E. Beyond the checklist: assessing understanding for HIV vaccine trial participation in South Africa. J Acquir Immune Defic Syndr 2006; 43:560-6.

59. World Medical Association. Declaration of Helsinki. Seoul: World Medical Association; 2008.

60. Council for International Organizations of Medical Sciences; World Health Organization. International ethical guidelines for biomedical research involving human subjects. Geneva: Council for International Organizations of Medical Sciences; World Health Organization; 1993.

Recebido em 09/Jul/2013

Versão final reapresentada em 23/Dez/2013

Aprovado em 06/Jan/2014 